Proceedings

\title{
Virtual Dimension Analysis of Hyperspectral Imaging to Char- acterize a Powder Sample from a Mine ${ }^{\dagger}$
}

\author{
Leonardo Chevez1, Alexander Apaza1, Jean Rodriguez1, Ruben Puga1 , Juan Dávalos2 and Héctor Loro1*
}

1 Af1 Facultad de Ciencias, Universidad Nacional de Ingeniería, Lima, Perú; hloro@uni.edu.pe

2 Instituto de Química-Física “Rocasolano"-CSIC, Madrid, Spain; jdavalos@csic.es

*hloro@uni.edu.pe

† Presented at the title, place, and date.

\begin{abstract}
Virtual Dimension (VD) procedure is used to analyze Hyperspectral Image (HIS) treatment-data in order to estimate the abundance of mineral components of a powder sample from a mine. Hiperspectral images coming from reflectance spectra (NIR region) are pre-treated using Standard Normal Variance (SNV) and Minimum Noise Fraction (MNF) methodologies. The endmember components are identified by the simplex growing algorithm (SVG) and after adjusted to the reflectance spectra of reference-databases using Simulated Annealing (SA) methodology. The obtained abundance of minerals of the sample studied is very near to the ones obtained using XRD with a total relative error of $2 \%$.
\end{abstract}

Keywords: Hyperspectral imaging, VD, SNV, MNF, SGA, XRD

\section{Introduction}

Hyperspectral imaging (HSI) technique has been successfully applied in different fields such as agriculture [1], environment [2], among others, and it also has been proved as a promising technology in the study of the earth-surface regarding its mineral composition [3]. Hyperspectral images consist of hundreds of contiguous and adjacent reflectance spectra of different areas of studied sample. The spectra cover bands ranging from Visible to Near Infrared (NIR) region, commonly between 0.3 to $2.5 \mu \mathrm{m}$ with bandwidth ranging between 2 and $10 \mathrm{~nm}$. The images can be monitored and collected by space-borne, air-borne, UAV-based sensors or simply in the laboratory using a moving platform with hyperspectral cameras. HIS is rapid and non-destructive technique that can provide quantitative information (abundances) of mineral composition of a sample, particularly of those containing carbonate minerals as calcite, aragonite and dolomite [4-9]. Hyperspectral images may be obtained from samples in any form: powders (as in our case), sands, broken, sawed or polished rock surfaces. The reflectance spectra recorded are yielded by a pure mineral or a mixture of several minerals. Additionally, these spectra depend also on the grain size of the minerals [10] and darkening effects such as spatial disintegration [11]. HSI technique allows to obtain a set of reflectance spectra for each pixel of the image, which when are correlated using adequate statistical tools, provide quantification of the pure mineral components present in the different parts of the studied sample (dust grains, in our case). This process is called "unmixing" process. It includes a pre-processing of the images followed by an identification of endmember pixels, which are a set of similar pixels present in the image under study. In general, the pixels provide information on the pure mineral components to be identified in the sample. There are several methods to obtain this information. In this work it has been used the Simulated Annealing (SA) method [12] which is an effective and general means of optimization inspired by metallurgy, where the temperature of a material determines its thermodynamic behavior. It has 
been successfully applied in the multi-hyperspectral remote sensing for classification [13- 1 14] and abundance estimation [15].

Materials and Experimental Methods

The sample studied is a white-powder collected from a mining zone in CajamarcaPerú. It was scanned using a benchtop hyperspectral imaging system HIS with a Pika NIR (Resonon Inc., Bozeman MT, USA) equipped with a $25 \mathrm{~mm}$ lens which has a spectral range of $900-1700 \mathrm{~nm}, 164$ spectral channels, $21.7^{\circ}$ field of view and a spectral resolution of 4.9 nm.

XRD analysis was performed with X-ray diffractometer PANalitycal model AERIS (Malvern Panalytical Ltda., Almedo, The Netherlands) $\mathrm{Cu}$-K $\alpha$ radiation $(\lambda=1.5406 \AA$, voltage $=40 \mathrm{kV}$ and current $=7.5 \mathrm{~mA}$ ). Scans were taken in the $2 \theta$ range $20^{\circ}$ to $90^{\circ}$ at increments of $0.02^{\circ}$. Rietvel analysis, using Highscore Plus Software, is carried out to obtain XRD spectra.

\section{HSI data analysis}

The process to obtain the unmixed reflectance-spectra data was as follows: Hyperspectral images, obtained in the NIR-spectra region of the sample, are pre-treatment using Standard Normal Variance (SNV) [16] and Minimum Noise Fraction (MNF) [17] methodologies. SNV is applied to normalize the data with respect to the different grain sizes of the sample. MNF transformation of the data is applied to diminish their dimensions. The goal of the MNF transform is to select components that maximize the signal-noise-ratio, leaving the components with the lowest ratio. To do this, the covariance matrices of both the recorded signal and the noise are constructed. There are several methods to estimate the noise covariance matrix. In this work is used the method suggested by Roger and Arnold [18-19]. The MNF provides a set of images ordered according to their quality and allows a more reliable identification and removal of noisy components, thus preserving components with useful information. The transformed data were used to identify the endmembers of the reflectance spectra which were done using Simplex Growing Algorithm (SGA) [2021]. A sub-pixel analysis is performed, in order to determine the mineral abundance in each endmember, using mineral database, through Simulated Annealing Linear Decomposition (SALD) adjustment. All these processes are within of Virtual Dimension (VD) methodology. The complete process is showed in the Figure 1. It is interesting to mention, that within a pixel there is a mixture of sub-pixels. The idea of sub-pixel mapping was first presented by Atkinson [22]. Since then, a series of techniques focused on better estimating the determination of fractional subpixel abundances has been proposed. In this work a sub-pixel decomposition of the endmembers is carried out, considering a superposition of reflectance spectra of the proposed minerals, in each endmember pixel. 


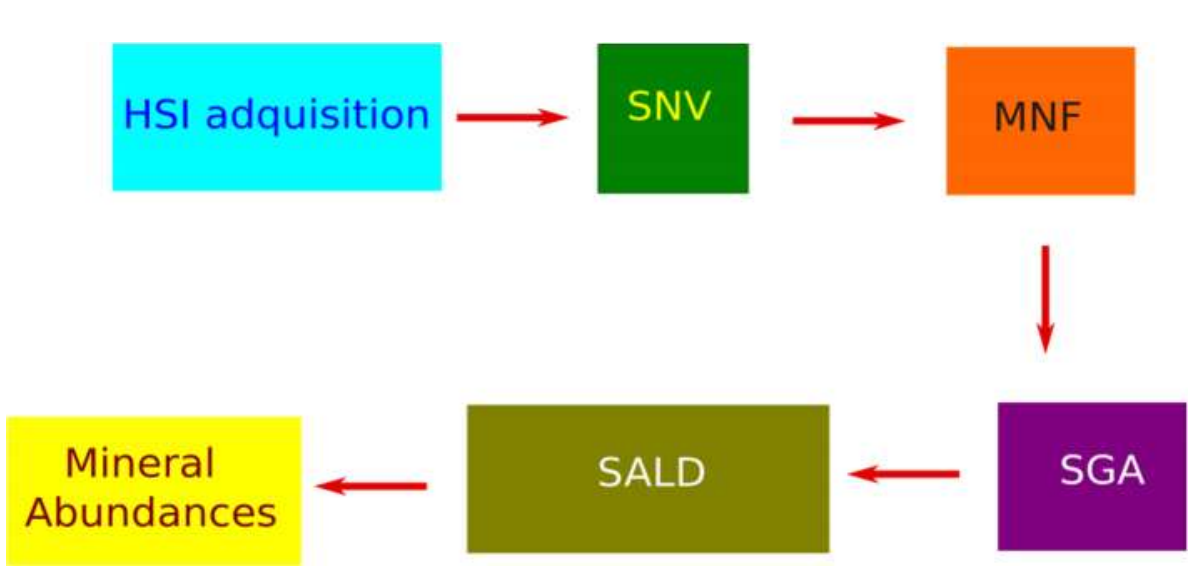

Figure 1. - Diagram of the sequence followed in this work to determine mineral abundance.

\section{Results XRD analysis}

XRD analysis is done to characterize of the sample studied and these will serve to validate HIS results. Figure 2 shows the XRD of the sample under study taken between $20^{\circ}$ and $90^{\circ}$. A Rietveld adjustment is used to identify the mineral components of the studied sample. The quality of the adjustment is given by noise-Rietveld registered in the Figure 2. The mineral components identified were Calcite $(86.6 \%)$, Dolomite $(9.9 \%)$ and Quartz (2.1\%). These results are used as reference set to compare the mineral abundance obtained by an HSI procedure. 


\section{HSI analysis}

As seen in the previous section, the analysis of the hyperspectral imagines data comprises several steps. The first step, includes a pre-processing treatment of the registered data using an algorithm (R software) implemented by our group, in followed by SNV and a MNF treatments (Fig. 1). After data pre-processing, the first task was to identify the Virtual Dimension (VD) of the transformed data, using eigenvalues and eigenvectors of the correlation matrix constructed with signal and noise data. The smallest eigenvalues correspond to eigenvectors or transformed bands that are less correlated and are associated with the white noise of the measurement. Figure 3 shows normalized eigenvalues of transformed MNF matrix data. This figure allows us to identify the uncorrelated endmembers which basically correspond to noise. It can be seen that after approximately the 15th component, there is no significant correlation value, so $\mathrm{VD}=15$ is an acceptable value that represents these data. The abundances calculated with this value are expected to be the closest to the reference values compared to those calculated with other VD values.

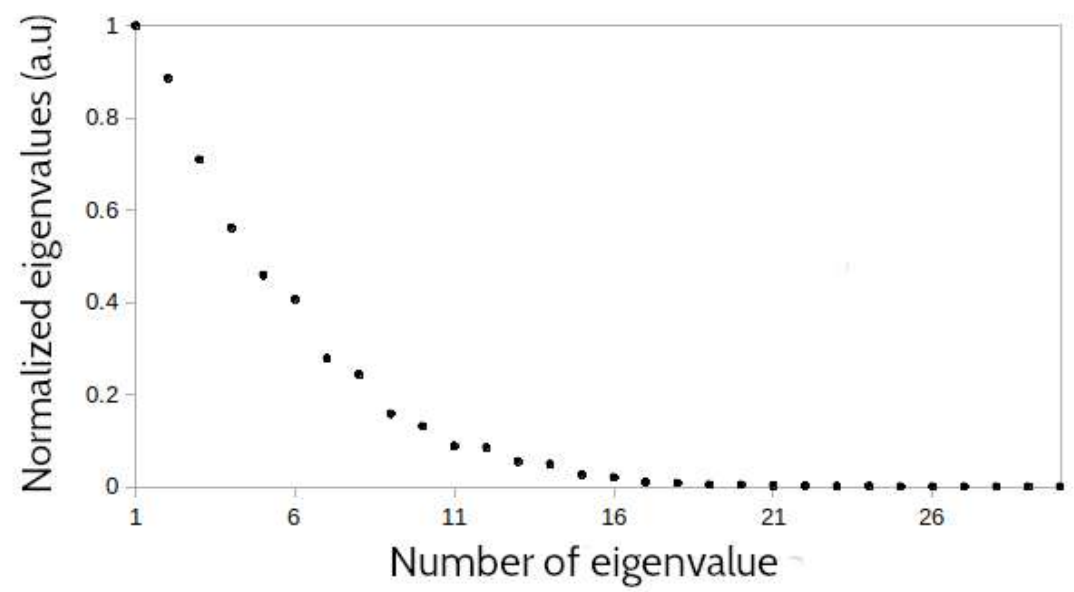

Figure 3. - Normalized eigenvalues of the transformed data using MNF method.

After data dimension reduction using $\mathrm{MNF}$, the endmembers pixels were searched by SGA, which allows not only to identify endmembers in the images, but also to estimate their abundances. To identify the endmembers, each pixel in the space formed by the eigenvectors of the MNF transformation is represented with a point. Each of these points corresponds to a pixel in the hyperspectral image. The endmembers in this new space define a volume that contains most of the pixels of the image. In order to identify the behavior of the endmembers obtained, the points associated with the endmembers were located in 2-D and 3-D graphics (figure 4) taking into account, respectively, the first two $\left[\mathrm{e}_{1}, \mathrm{e}_{2}\right]$ and three eigenvectors $\left[\mathrm{e}_{1}, \mathrm{e}_{2}, \mathrm{e}_{3}\right]$ ordered respect to their eigenvalues. The results obtained confirm the good behavior of the endmembers despite considering only some eigenvectors. 


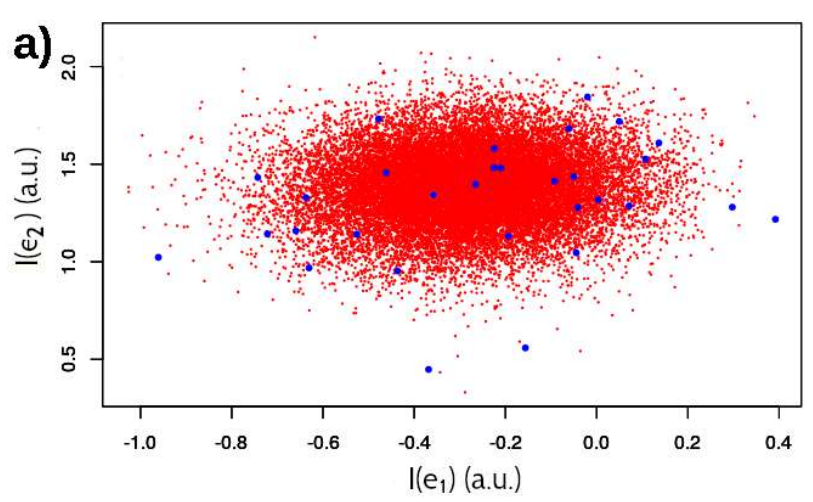

b)

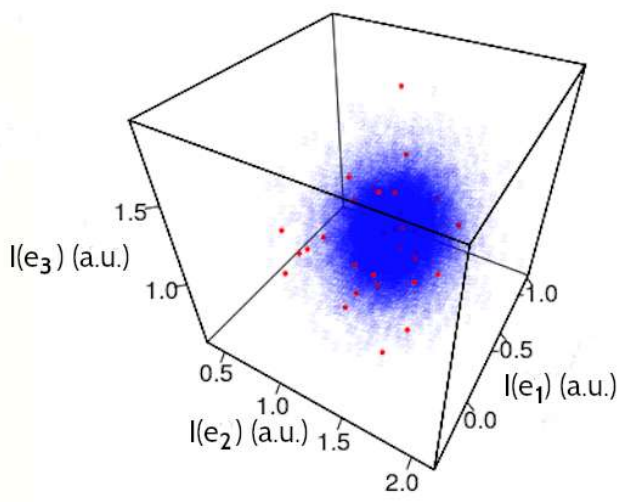

19

Figure 4. - Transformed data space points, (a) 2-D graphic), using [e1, $\left.\mathrm{e}_{2}\right]$ eigenvectors with the endmembers in blue and the rest of the pixels in red. (b) 3-D graphic, using [ $\mathrm{e}_{1}, \mathrm{e}_{2}, \mathrm{e}_{3}$ ] eigenvectors with the endmembers in blue and the rest of the pixels in red.

Once the endmembers have been identified, a sub-pixel analysis has been performed. Taking into account the minerals (Calcite, Dolomite and Quartz) identified by XRD (See Figure 2), their abundances are determined in each endmember of the sample. Reference reflectance spectra from the USGS database [24] were used for this analysis.

Each of the identified endmembers corresponds to a pixel in the initial hyperspectral image. The reflectance spectra of the pixels classified as endmembers are shown in Figure 5. The spectra of each of these endmembers were de-mixed using SALD. The final abundances have been determined considering the abundances of the endmembers in the sample image. 


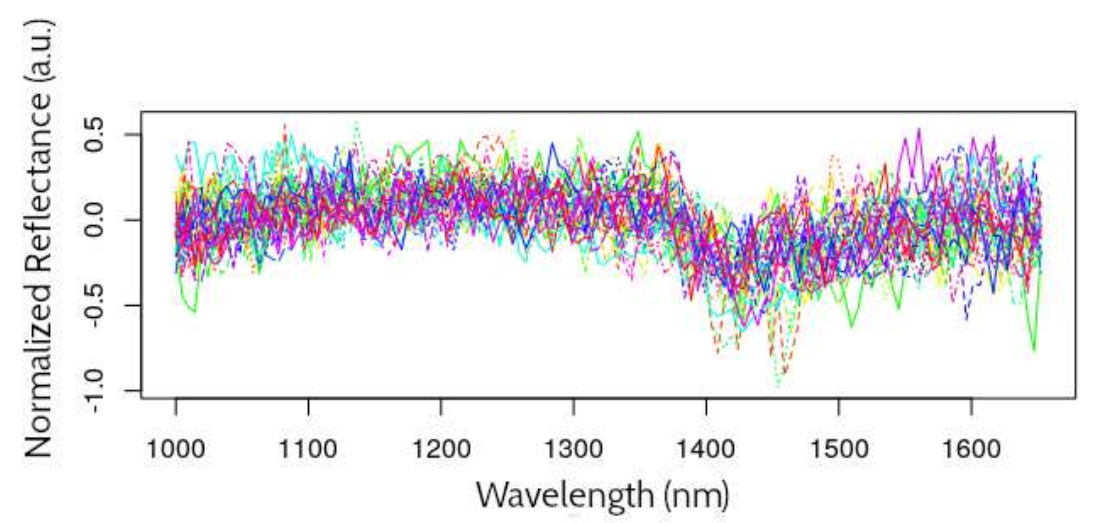

Figure 5. - Normalized reflectance spectra of pixels classified as endmembers.

This process was repeated considering a VD of 5, 10, 15, 20, 25 and 30, in order to corroborate the validity of considering, as significant eigenvalues, only the first 15 eigenvalues of the MNF transformation matrix. The final abundances obtained for these different $\mathrm{VD}$ values are shown in Table 1.

Table 1. - Calcite, dolomite and quartz abundances obtained by XRD and by an HSI analysis (considering $\mathrm{VD}=5,10,15,20,25,30)$.

\begin{tabular}{cccccccc}
\hline Component & XRD & VD=5 & VD=10 & VD=15 & VD=20 & VD=25 & VD=30 \\
\hline Calcite & 86.6 & 82.0 & 82.0 & 85.6 & 84.8 & 83.1 & 79.5 \\
\hline Dolomite & 9.9 & 11.0 & 10.3 & 8.4 & 8.4 & 9.4 & 11.5 \\
\hline Quartz & 2.1 & 7.0 & 7.7 & 6.0 & 6.9 & 7.5 & 9.0 \\
\hline Total relative error & --- & 2.50 & 2.76 & 2.02 & 2.45 & 2.66 & 3.53 \\
\hline
\end{tabular}

Table 1 shows that the lowest total relative error in the calculation of the abundances obtained by the HSI analysis is $2.02 \%$ and corresponds to $\mathrm{VD}=15$. This result is consistent with the most significant eigenvalues of the transformed MNF matrix. It is also interesting to mention that for $\mathrm{VD}=30$ there is a significant increase in the relative error, reaching $3.23 \%$, the highest of those calculated.

Finally, the Figure 6 shows the reflectance spectrum of endmember 6 and the spectrum adjusted using the final abundances found for $\mathrm{VD}=15$, showing a good correspondence between them. It is important to note that there is a significant variation in the spectrum around $1430 \mathrm{~nm}$. This variation has also been observed in the reference spectra used (USGS Database), a result that we consider as indicator of the good results obtained in this work despite the small range of spectral data used. 


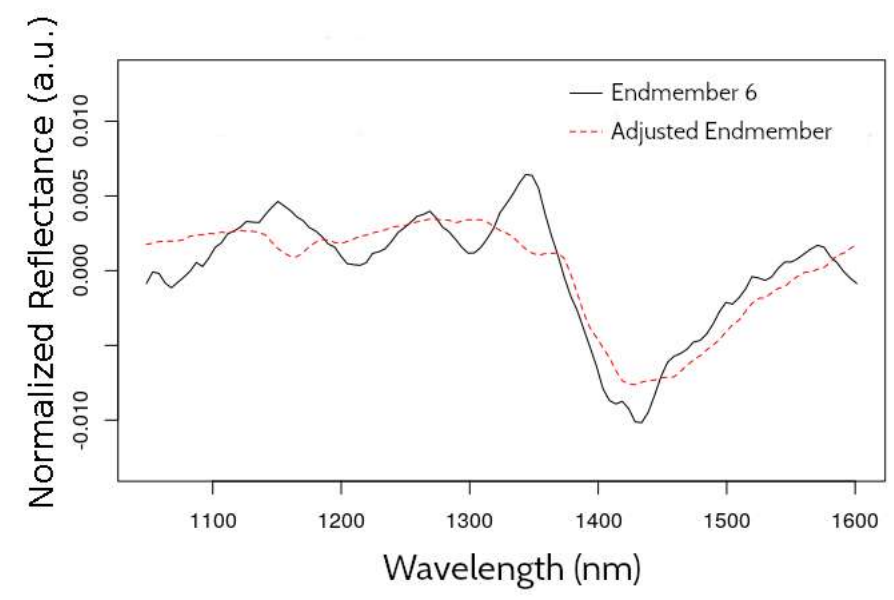

13

Figure 6. - Reflectance spectrum of endmember 6 and the spectrum adjusted using the final abundances found for $\mathrm{VD}=15$.

\section{Conclusions}

By means of an analysis of hyperspectral images (HSI technique), the abundance of mineral components of a sample of powdered carbonates from a Cajamarca-Peru mine was determined. HSI analysis includes processes of "unmixing" of images generated by reflectance spectra (NIR region) which are treated and transformed using SNV, MNF, SGA and SALD methodologies within the context of the Virtual Dimension (VD) procedure of transformed data (endmembers). It was found that for an optimal virtual dimension of $\mathrm{VD}=15$ the abundance of the mineral components of the sample is calcite $(85.6 \%)$, dolomite $(8.4 \%)$ and quartz $(6.0 \%)$ in excellent agreement with those determined by XRD since the error relative is $2.02 \%$.

\section{References}

[1] L. M. Dale, A. Thewis, C. Boudry, I. Rotar, P. Dardenne, V. Baeten, and J. A. Fernandez, “Hyperspectral Imaging Applications in Agriculture and Agro-Food Product Quality and Safety Control: A Review", Applied Spectroscopy Reviews, vol. 48, 2, 142-159, Jan , 2013.

[2] M. B. Stuart, A. J. S. McGonigle,and J. R. Willmott1, “Hyperspectral Imaging in Environmental Monitoring: A Review of Recent Developments and Technological Advances in Compact Field Deployable Systems", Sensors (Basel), vol. 19(14):3071, Jul, 2019.

[3] D. M. Rogge, B. Rivard, J. Zhang, and J. Feng, "Iterative Spectral Unmixing for Optimizing Per-Pixel Endmember Sets", IEEE Trans. Geosci. Remote Sens., vol. 44, no. 12, 3725-3736, Dec, 2006.

[4] J. B. Adams, "Visible and near-infrared diffuse reflectance spectra of pyroxenes as applied to remote sensing of solid objects in the solar system", J. Geophys. Res, vol. 79, 4829-4836, Nov, 1974.

[5] R. M. Hexter,R. “High-resolution, temperature-dependent spectra of calcite”, Spectrochim,Acta, vol. 10, 3, pp. 281-290, Jan, 1958.

[6] G. R. Hunt, and J. W. Salisbury, “Visible and near-infrared spectra of minerals and rocks: II Carbonates”, Mod. Geol., vol. 2, pp. 23-30, 1971.

[7] R. G. Burns, “Spectral mineralogy of terrestrial planets: scanning their surfaces remotely”. Mineral. Mag. 53, 135-151, April, 1989.

[8] S.. Tompkins, C. M. Pieters., "Spectral characteristics of lunar impact melts and inferred mineralogy", Meteorit. Planet. Sci., 45 (7), 1152-1169, 2010.

[9] B. Hapke,, "Theory of Reflectance and Emittance Spectroscopy”, 2nd Cambridge Univ. Press, New York, 2012. 
[10] T. Hiroi, C. M. Pieters, "Estimation of grain sizes and mixing ratios of fine powder mixtures of common geologic minerals", J. 1 Geophys. Res. Planets 99 (E5), 10,867-10,879, 1994.

[11] M. Pieters, E. M. Fischer, O. Rode, A. Basu, “Optical effects of space weathering the role of the finest fraction”, J. Geophys. Res. Planets 98, 20817-20824 (1993).

[12] B. S. Penn, "Using simulated annealing to obtain optimal linear end-member mixtures of hyperspectral data", Computers \& Geosciences 28, 809-817, Aug, 2002.

[13] A. Bardossy and L. Samaniego, "Fuzzy rule-based classification of remotely sensed imagery", IEEE Trans. Geosci. Remote Sens., vol. 2, no. 2, pp. 262-274, Mar, 2002.

[14] A. Robin, S. L. Hegarat-Mascle, and L. Moisan, "Unsupervised Subpixelic Classification Using Coarse-Resolution Time Series and Structural Information", IEEE Trans. Geosci. Remote Sens., vol. 46, no. 5, pp. 1359-1374, May 2008.

[15] P. Debba, E. Carranza, F. van der Meer, and A. Stein, “Abundances estimation of spectrally similar minerals by using derivative spectra in simulated annealing," IEEE Trans. Geosci. Remote Sens., vol. 44, no. 12, pp. 3469-3658, Dec. 2006.

[16] R. J. Barnes, M. S. Dhanoa and S.J. Lister, “Standard Normal Variate Transformation and De-trending of Near-Infrared Diffuse Reflectance Spectra", Appl. Spectrosc. 43, 5, 772-777, 1989.

[17] A. A. Green; M. Berman; P. Switzer; M. D. Craig, “A transform for ordering multispectral data in terms of image quality with implications for noise removal", IEEE Transactions on Geoscience and Remote Sensing, 26(1), p.65-74,1988.

[18] R. E. Roger, "Principal components transform with simple, automatic noise adjustrnent". Int. J. Remote Sens., vol. 17, no. 14, pp. 2719-2727, 1996.

[19] R. E. Roger and J. F. Arnold, “Reliably estimating the noise in AVIRIS hyperspectral images”, Int. J. Remote Sens., vol. 17, no. 10, pp. 1951-1962, 1996.

[20] C. Chang, C. Wu, C. Lo and M. Chang, "Real-Time Simplex Growing Algorithms for Hyperspectral Endmember Extraction", IEEE Transactions on Geoscience and Remote Sensing, vol. 48, no. 4, pp. 1834-1850, April 2010.

[21] P. Honeine and C. Richard, "Geometric Unmixing of Large Hyperspectral Images: A Barycentric Coordinate Approach", IEEE Transactions on Geoscience and Remote Sensing, vol. 50, no. 6, pp. 2185-2195, June 2012.

[22] P. Atkinson, "Innovations in GIS", vol. 4, ch. Mapping sub-pixel boundaries from remotely sensed images, New York Taylor and Francis, pp. 166-180, 1997.

[23] USGS Spectral Library Version 7: U.S. Geological Survey Data Series 1035, 61 p., https://doi.org/10.3133/ds1035.a. 